\title{
Correlation Length and Universality in the BCS-BEC Crossover for Energy-Dependent Resonance Superfluidity
}

\author{
S. Musolino ${ }^{1, a}$ and M.-L. Chiofalo ${ }^{2, b}$ \\ 1 Dept. of Physics "Enrico Fermi", Università di Pisa Largo Bruno Pontecorvo 3 I-56127 \\ Pisa, Italy \\ 2 Dept. of Physics "Enrico Fermi" and INFN, Università di Pisa Largo Bruno Pontecorvo \\ 3 I-56127 Pisa, Italy
}

\begin{abstract}
We consider the BCS-BEC crossover of a quantum Fermi gas at $T=0$ in the presence of an energy-dependent Fano-Feshbach resonance, driving the system from broad to narrow limits. We choose a minimal microscopic potential reproducing the two-particle resonance physics in terms of the scattering length $a$ and the effective range $R^{*}$ representing the resonance width, and solve the BCS mean-field equations varying $a, R^{*}$ and the density. We show that the condensate fraction manifests a universal behavior when the correlation length, measuring the pair size, is used as the crossover parameter. Generally, a negative effective range has the effect of stretching the crossover region between the two extreme regimes, as evidenced by the behavior of the chemical potential. These results can be useful in view of the more recent perspectives of realizing narrow resonances also by optical means and amenable as a base Quantum Monte Carlo simulations.
\end{abstract}

\section{Introduction}

The evolution of superfluidity in a quantum gas of interacting fermionic particles crossing over from a Bose-Einstein Condensation (BEC) $[1,2]$ of composite bosons to a Bardeen-Cooper-Schrieffer (BCS) [3] superfluidity of Cooper pairs is still an open problem. A number of theoretical and numerical efforts have been aimed to find universal behaviors after using different classes of interaction potentials whose strength might drive the crossover [4]. The seminal works of Eagles [5], Leggett [6] and Nozières and Schmitt-Rink [7] have introduced the concept of crossover physics in the context of superconductivity and superfluidity: they predicted how observables like the chemical potential and the fraction of Bose-Einstein condensed pairs would smoothly evolve their behaviors from a BCS-like state of Cooper pairs to a BEC-like state of bosons composed by two fermions with varying the attractive interaction.

The smooth evolution in the BCS-BEC crossover has become a hot topic in the context of high-temperature superconductivity, also hinging on the celebrated log-log

\footnotetext{
a e-mail: sil.musolino@gmail.com

b e-mail: maria.luisa.chiofalo@unipi.it
} 
plot by Uemura et al. [8], showing that the measured critical temperatures of several fermionic and bosonic systems can be collected to stay along approximately the same line as a function of an effective Fermi temperature $T_{F}$, with high- $T_{C}$ superconductors in between the BCS and BEC limits. This unique description independent of the compounds details, has boosted further theoretical investigations, especially the work of Pistolesi and Strinati [9]. They demonstrated that such a universal behavior, that is independent of the details of the interaction potential and of the single-particle density of states, can be described using the correlation length, representing the size of the Cooper pair evolving into bosonic molecules. However, high- $T_{C}$ superconductors are complex systems, where microscopic details are hard to control and disentangle. The realization of superfluidity in ultracold bosonic [10-13] and fermionic [14-16] quantum gases is instead characterized by the conditions of extreme precision and control typical of atomic physics, with the possibility of tuning temperature, dimensionality, and interactions in strength, sign and range. Therefore, they represent a very convenient experimental and theoretical laboratory to probe the crossover idea and link it to microscopic models. In particular, the strength and sign of interactions can be controlled through the mechanism of Fano-Feshbach resonances [17,18], where the energy of a bound state of the interatomic potential composed by two (fermionic) atoms, can be magnetically or optically tuned at resonance with the energy of a scattering state of the same interatomic potential where the two atoms are free [19]. At low energy, the whole mechanism can be described by two relevant length scales: the scattering length, tunable from negative to positive large values, and the effective range of the potential, related to the resonance width. The latter has to be compared to the background scattering length (i.e. away from resonance) in two-body physics, and to the interparticle spacing in the many-body case [20,21]. For broad resonances the effective range becomes immaterial. A third relevant length is the interparticle spacing.

Two questions are still open. First, whether a unique microscopic model exists that could describe the crossover for both broad and narrow resonances. This is becoming a timely issue with the more recent possibility of realizing narrow resonances by optical means [19]. Theories are usually classified in two- and single- channel Hamiltonians $[16,22]$, the channels representing the scattering and bound states contributing to the resonance mechanism. While the two-channel Hamiltonians stem from the original Fano-Feshbach idea $[23,24]$ and provide a natural theoretical framework, singlechannel theories have been often used to describe the broad-resonance case. This is presently very well understood also beyond mean-field by theoretical [25] and Quantum Monte Carlo (QMC) methods [26]. Second question is whether the crossover can be described by only one universal parameter. The usual statement is found in literature, that no universal behavior can be observed in the narrow resonance case [22]. Here, we dig deeper into this last question, after considering the BCS-BEC crossover of a quantum Fermi gas at zero temperature in the presence of an energy-dependent Fano-Feshbach resonance, driving the system from broad to narrow limits. We find that the physics is effectively described in terms of three physical parameters, that are scattering length $a$, effective range $R^{*}$, and density $n$, one more with respect to the class of potentials considered in $[7,9]$. We show that the condensate fraction manifests a universal behavior when the correlation length, as first introduced in [9], is used as the only crossover parameter, with all the data sets collapsing into one single curve (see Fig. 6). In addition, we find that a negative effective range has the overall effect of stretching the crossover region between the two extreme regimes. For the chemical potential, this actually makes the universal behavior less evident with respect to the striking result obtained in [9] with a two-parameter model potential and in a wider density range. To this aim, we use a single-channel Hamiltonian, able to reproduce the two-body resonance physics, with the interaction potential in the 
form of a well-barrier model $[27,28]$. The choice of the single-channel Hamiltonian has been performed in view of using the present mean-field results as starting point for future QMC studies, extending the single-channel analysis for broad resonances [26] and for energy-dependent resonances limited to positive $R^{*}$ [29]. On the other hand, we discuss limits of the present well-barrier model in the deep BEC regime.

The paper is organized as follows. Sect. 2 introduces the model. Sect. 3 describes the mean-field equations and defines the observables. The results are discussed in Sect. 4, and summarized in Sect. 5 along with conclusive remarks and future perspectives.

\section{The model}

As discussed in the introduction, we use the standard BCS formulation for the Hamiltonian of a system with $N$ fermions of mass $m$ equally distributed in two spin states [3]:

$$
\hat{H}=\sum_{\mathbf{k}} t_{\mathbf{k}} c_{\mathbf{k} \sigma}^{+} c_{\mathbf{k} \sigma}+\sum_{\mathbf{k k}^{\prime} \mathbf{q}} V_{\mathbf{k k}^{\prime}} c_{\mathbf{k}+\mathbf{q} / 2 \uparrow}^{+} c_{-\mathbf{k}+\mathbf{q} / 2 \downarrow}^{+} c_{-\mathbf{k}^{\prime}+\mathbf{q} / 2 \downarrow} c_{\mathbf{k}^{\prime}+\mathbf{q} / 2 \uparrow}
$$

where $\hat{c}_{\mathbf{k} \sigma}$ are the fermionic operators, $t_{\mathbf{k}}=\hbar^{2} k^{2} / 2 m$ is the free-fermion dispersion relation. $V_{\mathbf{k k}^{\prime}}$ is the interaction potential representing an effective interaction among the particles, that here is wanted to reproduce the two-body physics of the energydependent Fano-Feshbach two-channel mechanism.

As enlightened in [30], it is in principle possible to reduce a two-channel into a singlechannel model. This is appropriate when a minimal number of microscopic parameters describe the relevant two-body physics, to which the many-particle results reduce to. For the resonance mechanism under consideration, three parameters may be seen to characterize the low-energy scattering: the scattering length $a_{b g}$ away from resonance, the detuning $\nu$ from resonance and the resonance width. For a magnetically-induced resonance for example, the two channels are coupled via an exchange interaction $g$ determining the resonance width, $\nu$ is the energy difference between the bound state of the closed channel and the threshold of the continuum in the open channel. These quantities are related to the scattering length $a$ via $a=a_{b g}-m|g|^{2} /\left(4 \pi \hbar^{2} \nu\right)$. The coupling $g$ defines a characteristic length $R^{*}=-8 \pi \hbar^{4} /\left(m^{2}|g|^{2}\right)$, that is the effective range [30]. Its meaning appears from the expression of the effective inverse scattering phase shift, which to second order in $k$ can be expressed as [22]

$$
\frac{1}{a_{e f f}(k)} \simeq \frac{1}{a}-\frac{1}{2} R^{*} k^{2}
$$

In an equivalent single-channel model, an appropriate $V_{\mathbf{k}, \mathbf{k}^{\prime}}$ is to be devised to reproduce $a$ and $R^{*}$. Following [27-29], we take it in real space in the simplest form of a short-range square well, followed by a repulsive barrier:

$$
V(r)= \begin{cases}-V_{0}, & \text { if } r<r_{0}, \\ V_{1}, & \text { if } r_{0} \leq r<r_{1}, \\ 0, & \text { otherwise. }\end{cases}
$$

This is depicted in Fig. 1. In essence, the well-barrier depth $V_{0}+V_{1}$ determines $a$, and the barrier width $r_{1}-r_{0}$ drives $R^{*}$ and the resonance width. $V_{0}, r_{0}, V_{1}$, and $r_{1}$ can be appropriately connected to the scattering theory, after solving the two-body problem. Closely following the pedagogical work by Jensen et al. [28], $a$ and $R^{*}$ can 


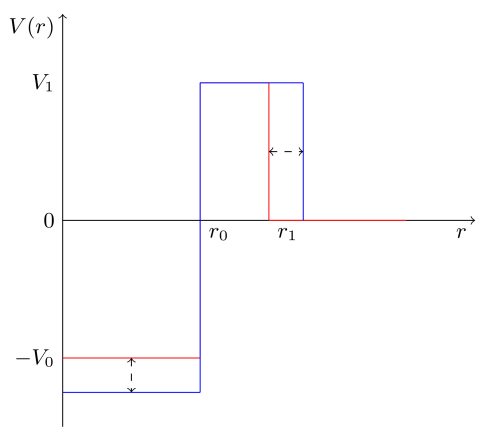

Fig. 1. Square well-barrier model reproducing the physics of an energy-dependent FanoFeshbach resonance. The two physical parameters, $a$ and $R^{*}$ are determined after solving the 2-particle scattering problem: in essence $V_{0}+V_{1}$ determines $a$ and $r_{1}-r_{0}$ determines $R^{*}$.

be cast in the form:

$$
\begin{aligned}
a & =r_{1}-\frac{k_{1} \alpha+k_{0} \beta}{k_{1}\left(k_{0}+k_{1} \alpha \beta\right)}, \\
R^{*} & =r_{1}-\frac{k_{0}^{2}+k_{1}^{2}}{a \gamma} \frac{1}{k_{0} k_{1}}\left[1+\frac{k_{0} k_{1} r_{0}\left(1-\beta^{2}\right)}{a \gamma}-\frac{r_{1}}{a}+\frac{\beta}{k_{1} a}\right]+\frac{1}{k_{1}^{2} a}-\frac{r_{1}^{3}}{a^{2}},
\end{aligned}
$$

where $k_{1}=\sqrt{m V_{1} / h^{2}}, k_{0}=\sqrt{m V_{0} / h^{2}}, \alpha \equiv \tan \left(k_{0} r_{0}\right), \beta \equiv \tanh \left(k_{1}\left(r_{1}-r_{0}\right)\right)$, and $\gamma \equiv k_{1}\left(k_{0}+k_{1} \alpha \beta\right)$. It is possible to check by inspection that $R^{*} \leqslant r_{1}$ always.

In the actual determination of $V(r)$, we first fix $V_{1}$ to stay within the first resonance. We then fix $r_{0}$ in order to provide the wanted diluteness parameter $n r_{0}^{3}$ at given density. Finally, given $a$ and $R^{*}$ we determine the remaining parameters $V_{0}$ and $r_{1}$. Notice that at variance with [28], where $a$ and the effective range at resonance $R_{0}^{*}$ are varied, we have found more convenient to vary $a$ and $R^{*}$ : this allowed us to compare with the two-channel model results existing in literature.

\section{Mean-field approach at zero temperature}

Closely following the BCS derivation [31], we consider the trial wave function $\left|\Phi_{B C S}\right\rangle=$ $\prod_{\mathbf{k}}\left(u_{\mathbf{k}}+v_{\mathbf{k}} \hat{c}_{\mathbf{k} \uparrow}^{+} \hat{c}_{-\mathbf{k} \downarrow}^{+}\right)|0\rangle$, representing a collection of pairs of fermions created in the vacuum $|0\rangle$, where the two fermions have opposite spins and correlated momenta $+\mathbf{k}$ and $-\mathbf{k}$, their total momentum being $\mathbf{q}=0$. Here, $u_{\mathbf{k}}$ and $v_{\mathbf{k}}$ are $\mathbf{k}$-dependent functions that satisfy the normalization condition $\left|u_{\mathbf{k}}\right|^{2}+\left|v_{\mathbf{k}}\right|^{2}=1$. Minimization of the free-energy $\hat{F}=\hat{H}-\mu \hat{N}$, with $\mu$ the chemical potential, yields:

$$
u_{\mathbf{k}}^{2}=\frac{1}{2}\left(1+\frac{\epsilon_{\mathbf{k}}}{E_{\mathbf{k}}}\right), v_{\mathbf{k}}^{2}=\frac{1}{2}\left(1-\frac{\epsilon_{\mathbf{k}}}{E_{\mathbf{k}}}\right) .
$$

Here, $E_{\mathbf{k}}=\sqrt{\epsilon_{\mathbf{k}}^{2}+\Delta_{\mathbf{k}}^{2}}$ is the excitation energy, $\Delta_{\mathbf{k}}$ the gap function, and $\epsilon_{\mathbf{k}}$ the single-particle energy that in our choice includes the Hartree-Fock contribution. The 
well-known self-consistent equations result to be:

$$
\begin{aligned}
\Delta_{\mathbf{k}} & =-\sum_{\mathbf{k}} V_{\mathbf{k}, \mathbf{k}^{\prime}} \frac{\Delta_{\mathbf{k}^{\prime}}}{2 E_{\mathbf{k}^{\prime}}}, \\
\epsilon_{\mathbf{k}} & =t_{\mathbf{k}}-\mu+\frac{1}{2} V_{H} n-\sum_{\mathbf{k}^{\prime}} V_{\mathbf{k}, \mathbf{k}^{\prime}}\left(1-\frac{\epsilon_{\mathbf{k}^{\prime}}}{E_{\mathbf{k}^{\prime}}}\right), \\
N & =2 \sum_{\mathbf{k}} n_{\mathbf{k}}=2 \sum_{\mathbf{k}}\left|v_{\mathbf{k}}\right|^{2}=\sum_{\mathbf{k}}\left(1-\frac{\epsilon_{\mathbf{k}}}{E_{\mathbf{k}}}\right),
\end{aligned}
$$

where $V_{\mathbf{k}, \mathbf{k}^{\prime}}$ is the Fourier transform of (3) and $V_{H}=4 \pi\left[V_{1}\left(r_{1}^{3}-r_{0}^{3}\right)-V_{0} r_{0}^{3}\right] / 3$ is the Hartree contribution. Considering only the s-wave scattering contribution in this low-energy regime, one has:

$$
\begin{aligned}
V\left(k, k^{\prime}\right) & =\frac{2 \pi}{k k^{\prime}}\left[\left(V_{0}+V_{1}\right)\left(\frac{\sin \left(\left(k+k^{\prime}\right) r_{0}\right)}{k+k^{\prime}}-\frac{\sin \left(\left(k-k^{\prime}\right) r_{0}\right)}{k-k^{\prime}}\right)+\right. \\
& \left.-V_{1}\left(\frac{\sin \left(\left(k+k^{\prime}\right) r_{1}\right)}{k+k^{\prime}}-\frac{\sin \left(\left(k-k^{\prime}\right) r_{1}\right)}{k-k^{\prime}}\right)\right]
\end{aligned}
$$

Eqs. (7)-(8) are non-linear and self-consistent expressions, to be solved numerically by iteration in order to provide $\Delta(k), E(k)$ and $\mu$. In particular, after iterating (7) and (8), from initial guesses for $\Delta(k)$ and $\epsilon(k)$, the corresponding value of $\mu$ is found as a root of Eq. (9). The procedure is repeated until full convergence.

We conclude this section by listing all the observables that are calculated and illustrated in the next one. First, the condensate fraction $N_{0}$, given by [31]

$$
N_{0}=\sum_{\mathbf{k}}\left|u_{\mathbf{k}} v_{\mathbf{k}}\right|^{2} .
$$

The correlation length $\xi$ represents a crucial quantity for our results. Therefore, we first calculate the pair correlation function $g_{\sigma, \sigma^{\prime}}(\mathbf{r})[32]$, with $\sigma=\uparrow, \downarrow$. Because of spin symmetry, one has $g_{p}(\mathbf{r})=2 g_{\uparrow \uparrow}(\mathbf{r})=2 g_{\downarrow \downarrow}(\mathbf{r})$ for the parallel contribution and $g_{a}(\mathbf{r})=$ $2 g_{\uparrow \downarrow}(\mathbf{r})=2 g_{\downarrow \uparrow}(\mathbf{r})$ for the antiparallel one. The correlation length $\xi$ is a measure of the variance of $g_{\uparrow \downarrow}(\mathbf{r})$ and embodies all the interaction effects. After performing the angular integration and conveniently expressing in units of Fermi wave-vector $k_{F}$, one has:

$$
\left(k_{F} \xi\right)^{2}=\frac{\int d^{3} r r^{2}\left(g_{\uparrow \downarrow}(r)-1\right)}{\int d^{3} r\left(g_{\uparrow \downarrow}(r)-1\right)}=\frac{\int_{0}^{\infty} d k k^{2}\left|\frac{d}{d k}(u(k) v(k))\right|^{2}}{\int_{0}^{\infty} d k k^{2}|u(k) v(k)|^{2}},
$$

where

$$
g_{\uparrow \downarrow}(r)=1+9\left|\int_{0}^{\infty} d k \frac{k}{r} \sin (k r) u(k) v(k)\right|^{2}
$$

For completeness, $g_{\uparrow \uparrow}(r)=1-\left.9\left|\int_{0}^{\infty} d k k \sin (k r)\right| v(k)\right|^{2} /\left.r\right|^{2}$.

\section{Results}

Before illustrating the results we define our parameter space. Tab. 1 summarizes all the length scales of the problem, with the corresponding range of values here adopted. Notice that all the microscopic potential length scales, $r_{0}, r_{1}, k_{0}^{-1}$ and $k_{1}^{-1}$ are smaller than the interparticle spacing $\left(n / k_{F}^{3}\right)^{-\frac{1}{3}}$, while $k_{F} a$ and possibly $k_{F} R^{*}$ may diverge. We recall that $\left(k_{F} a\right)^{-1}<0$ and $\left(k_{F} a\right)^{-1}>0$ correspond to BCS and BEC sides of 
Table 1. Length scales of the model and values of $a$ and $R^{*}$ which they determine (see text). Notice that $r_{0}, r_{1}, k_{0}^{-1}$, and $k_{1}^{-1}<\left(n / k_{F}^{3}\right)^{-\frac{1}{3}}$, while $k_{F} a$ and $k_{F} R^{*}$ may diverge.

\begin{tabular}{lllllll}
\hline$k_{F} r_{0}$ & $k_{F} r_{1}$ & $\left(k_{0} / k_{F}\right)^{-1}$ & $\left(k_{1} / k_{F}\right)^{-1}$ & $\left(n / k_{F}^{3}\right)^{-\frac{1}{3}}$ & $k_{F} R^{*}$ & $\left(k_{F} a\right)^{-1}$ \\
\hline 0.31 & $\sim(0.35,0.7)$ & $\sim(0.1,0.2)$ & 0.15 & $\left(3 \pi^{2}\right)^{\frac{1}{3}}=3.1$ & $(0.2,-0.75)$ & $(-10,+2)$ \\
\hline
\end{tabular}

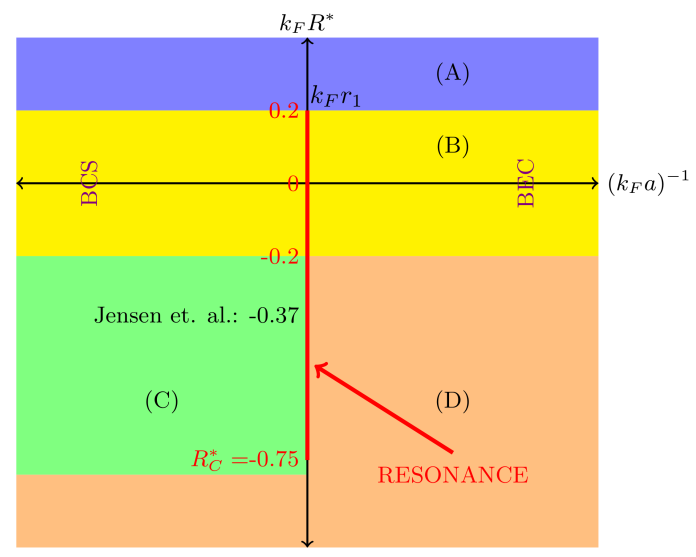

Fig. 2. Parameter space for $n r_{0}^{3}=10^{-3}$. The regions (A)-(D) are discussed in the text.

the resonance, respectively, with $\left(k_{F} a\right)^{-1}=0$ marking resonance. As to $R^{*}$, Eq. (2) provides a useful reading key for the whole discussion below: $R^{*}<0$ values make $a_{\text {eff }}^{-1}$ more positive and therefore move the system towards a more BEC character, similarly $R^{*}>0$ values drive a more BCS nature.

Fig. 2 represents the parameter space for the case with density $n$ such that $n r_{0}^{3}=$ $10^{-3}$. Four relevant regions (A)-(D) are evidenced. Region (A) is less interesting when compared with the two-channel model where $R^{*}<0$ and is not accessible in the wellbarrier model where $R^{*}<r_{1}$. Region (B) is the main concern of this work, with (C) corresponding to a trivial extension of it. Finally, we have found that region (D) in the extreme boson-like regime appears to be hardly accessible to the well-barrier model. On physical grounds, this might be due to extreme localization of the pair wavefunction in the deep BEC regime, which hampers condensation at $k=0$. If this were the case, we should expect that the largest accessible $R^{*}$ value, say $R_{C}^{*}$, be dictated by the condition $R_{C}^{*} \sim 2 r_{0}$. Indeed, a negative value of the effective range $R_{0}^{*}$ at resonance implies that a large portion of the pair density be inside the well with $R_{0}^{*} \lesssim 2 r_{0}$ [28]. In practice, we have numerically checked this hypothesis by exploring the largest accessible $R^{*}$ value for different $k_{F} r_{0}$, as reported in Tab. 2, where the trend $R_{C}^{*} \sim 2 r_{0}$ seems to emerge. The numerical check has been repeated by using two different strategies to solve the gap equation, namely iteration and Newton methods. This limit has not been discussed in [28].

Besides $a$ and $R^{*}$, the density is varied as well. The largest negative $k_{F} R^{*}=-3.6$ is attainable at $n r_{0}^{3}=0.125$. Here, the resonance can be viewed as narrow in the many-body sense [21]. Notice that too large values of $n r_{0}^{3}$ worsen the validity of a mean-field approach, making QMC studies even more necessary. 
Table 2. Lowest critical value $R_{C}^{*}$ of $R^{*}$ for different densities $n r_{0}^{3}$ (see the main text).

\begin{tabular}{llll}
\hline$n r_{0}^{3}$ & $k_{F} r_{0}$ & $\left(k_{F} r_{0}\right)^{-1}$ & $k_{F} R_{C}^{*}$ \\
\hline 0.125 & 1.54 & 0.65 & -3.6 \\
$10^{-3}$ & 0.31 & 3.23 & -0.75 \\
$10^{-4}$ & 0.14 & 7.14 & -0.2 \\
$10^{-5}$ & 0.07 & 14.28 & -0.05 \\
\hline
\end{tabular}

As the numerical parameters in the self-consistent procedure, we have used up to 1200 points dividing the integration in three intervals to account for the different length scales of the problem, and treating the last integral with the variable change $k \rightarrow$ $k^{-1}$. A typical convergence procedure required from tens to hundreds iterations for $\Delta(k)$ and $\epsilon(k)$ depending on the position in the parameter space. For each calculated quantity, we have estimated the error bar by varying all the different physical and algorithm parameters, and observed the error propagation. In the following figures, the size of the used symbols represents the uncertainty of the data.

\subsection{Gap function}

For completeness, we first report results already known from [28], though in an extended parameter region. Fig. 3 displays $\Delta(k)$ for $k_{F} R^{*}$ and $\left(k_{F} a\right)^{-1}$ values reported in the legends. We first notice the periodicity character, that is dictated by $2\left(k_{F} r_{0}\right)^{-1}$, as evident from panel (d) where two different $k_{F} r_{0}$ cases are compared. Second, panel (a) evidences how the BEC character manifests as a bump at finite $k / k_{F}$ value, corresponding to building up of the pair wave function in the center of the well at $k_{F} r_{0} / 2$. The bump is lost on the BCS side of the resonance, where the gap maximum moves towards $k=0$, as expected. In panel (b), the effect of $R^{*}>0$ values is evident, always driving the system towards a BCS-like nature. Finally, panel (c) illustrates the effect of $R^{*}<0$ values in enhancing the bump size and therefore the BEC character. While in the BCS regime $\Delta(k=0)$ clearly is the significant parameter, on the BEC side $\Delta(0)$ is unchanged and the bump size increased, so that $\int d^{3} k|\Delta(k)|^{2}$ is the relevant parameter, evaluating the probability for the pair to be located within the well.

\subsection{Correlation length}

Fig. 4 illustrates the behavior of the correlation length $k_{F} \xi$ as a function of $\left(k_{F} a\right)^{-1}$ and for different values of $k_{F} R^{*}$. The inset displays the typical antiparallel pair correlation function $g_{\uparrow \downarrow}(r)$ at different $\left(k_{F} a\right)^{-1}$ values, from which $k_{F} \xi$ is computed via (12). It is evident the usefulness of $k_{F} \xi$ as a parameter characterizing the crossover. In fact, $k_{F} \xi$ progressively increases while $\left(k_{f} a\right)^{-1}$ becomes large and negative and $k_{F} R^{*}>0$ : we have checked that $k_{F} \xi \rightarrow k_{F} \xi_{\text {Pippard }}$, with the Pippard coherence length $\xi_{\text {Pippard }}=\hbar^{2} k_{F} /(m \Delta)$, where $\Delta$ is the BCS-gap parameter. On the BEC side with $\left(k_{f} a\right)^{-1}$ large and positive and $k_{F} R^{*}<0$, we see that $k_{F} \xi \rightarrow k_{F} r_{0}$ (dashed horizontal line), as expected. Similar results hold at the other density values.

\subsection{Universal behaviors}

We are now in a position to discuss the main results of this work. Fig. 5 shows $\mu / E_{F}$ versus $k_{F} \xi$. Following the original idea [9], we use the following normalization: $\mu / E_{F}^{*}$ 


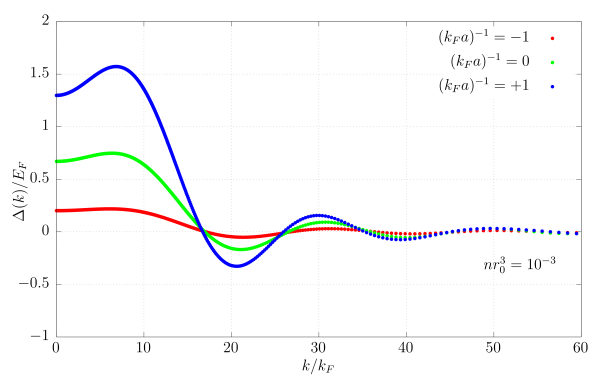

(a) $k_{F} R^{*}=0$.

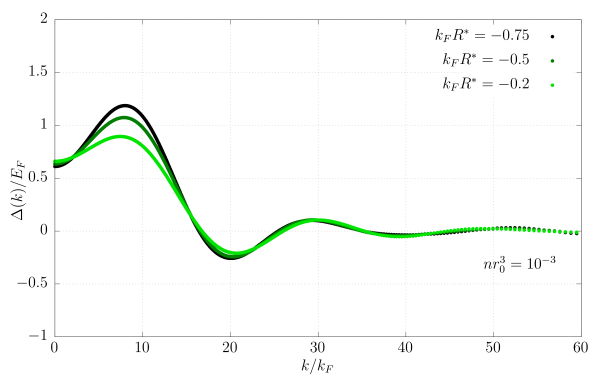

(c) $\left(k_{F} a\right)^{-1}=0, k_{F} R^{*}<0$.

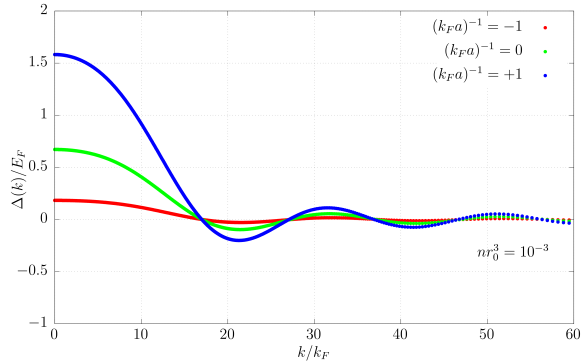

(b) $k_{F} R^{*}=0.2$.

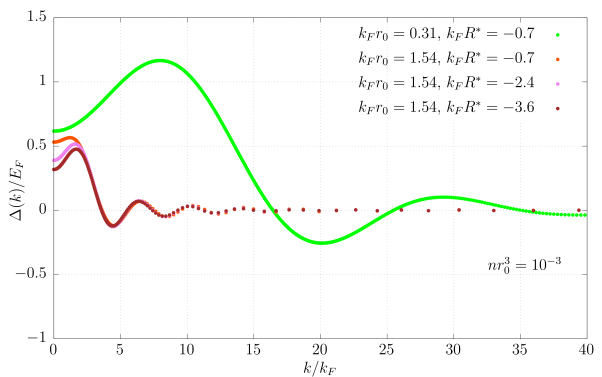

(d) $\left(k_{F} a\right)^{-1}=0$.

Fig. 3. Gap function $\Delta(k)$ in units of $E_{F}=\hbar^{2} k_{F}^{2} / 2 m$ vs. $k$ in units of $k_{F}$, for different values of $\left(k_{F} a\right)^{-1}, k_{F} R^{*}$ and $n r_{0}^{3}$ (or $k_{F} r_{0}$ ), as in legend.

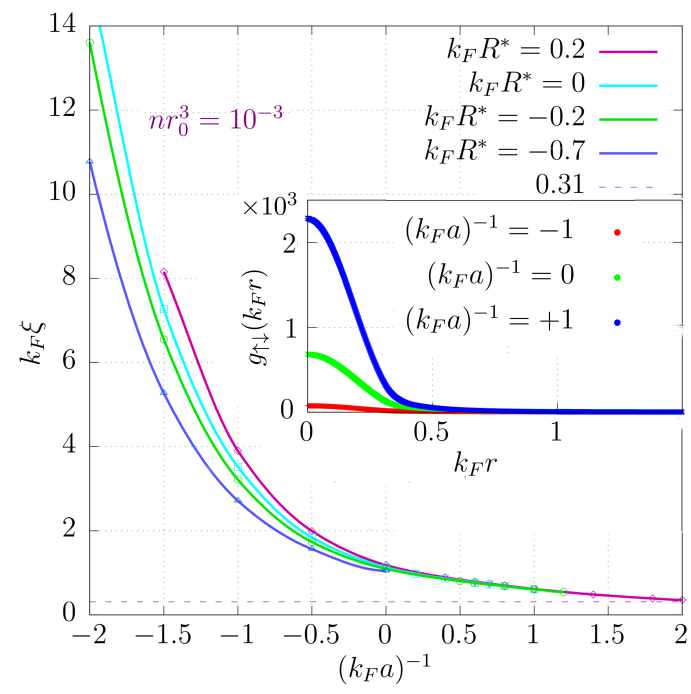

Fig. 4. Correlation length $k_{F} \xi$ versus the parameter $\left(k_{F} a\right)^{-1}$ for different values of $k_{F} R^{*}$ as in the legend and $n r_{0}^{3}=10^{-3}$. The dashed line marks the value $k_{F} r_{0}$ (see text). Inset: $g_{\uparrow \downarrow}(r)$ for $\left(k_{F} a\right)^{-1}$ in the legend and $k_{F} R^{*}=0$. 


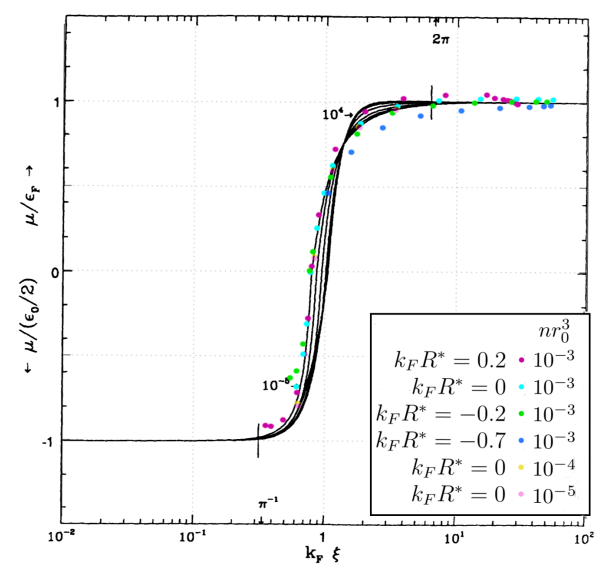

Fig. 5. Chemical potential $\mu$ vs. correlation length $k_{F} \xi$. $\mu$ is normalized to $E_{F}$ when $\mu>0$ (BCS side) and to half the binding energy $E_{b} / 2$ when $\mu<0$. Solid lines are extracted from [9]. Points: this work for the model parameters $\left(k_{F} a\right)^{-1}, k_{F} R^{*}$ and $n r_{0}^{3}$ as in the legend.

when $\mu>0$, with $E_{F}^{*}=\hbar^{2} k_{F}^{2} /\left(2 m^{*}\right)$ in terms of the effective mass determined from $\epsilon(k)$, and $\mu /\left(E_{b} / 2\right)$ when $\mu<0$, with $E_{b} / 2$ the binding energy for two particles in the well-barrier model. This is given by $E_{b} /\left(2 E_{F}\right)=2[\zeta-1+\sqrt{1-2 \zeta}] /\left(k_{F} R^{*}\right)^{2}[22]$ at given $R^{*}, a$, and their ratio $\zeta \equiv\left(k_{F} R^{*}\right) /\left(k_{F} a\right)$.

We have explicitly collected all our data sets at different $k_{F} R^{*}$ and $n r_{0}^{3}$ as in the legend and superimposed them on top of the original graph in [9]. In [9], the microscopic potential is a separable one, described by two parameters, i.e. scattering length and density. The different solid lines from the original graph refer to different values of $n / k_{0}^{3}$, with $k_{0}^{-1}$ being the characteristic wave vector of the model interaction potential [9]. It is seen that for small $k_{F} \xi<\pi^{-1}$ and large $k_{F} \xi>2 \pi$, all the curves collapse to either -1 or +1 . In our case, the same idea is extended to analyze $\mu$ in terms of the three parameters of the present theory: $\left(k_{F} a\right)^{-1}, k_{F} R^{*}$ and $n r_{0}^{3}$. We find a similar behavior, though in a smaller density range, with two special remarks. First, $R^{*}$ has the effect of stretching the crossover region between the two extreme regimes, in which the data are scattered. Therefore, while $k_{F} \xi$ demonstrates to be a suited parameter to describe the crossover, the degree of universal behavior reduces with respect to the striking result obtained in [9]. Second, in our case with $R^{*}<0$, the deep BEC regime is moved toward the leftmost side of the graph, which is not accessible due to the already discussed model limitations in region (D), Fig. 2.

The universal behavior is striking when the normalized condensate fraction $2 N_{0} / N$ is considered. This is displayed in Fig. 6 as function of $k_{F} \xi$ for our data sets. All data sets collapse in only one curve with the expected limiting values $2 N_{0} / N \rightarrow 1$ on the BEC side, and $2 N_{0} / N \rightarrow \Delta(k=0) / E_{F}$ on the BCS regime. While the chemical potential appears to be more sensitive to the details of the interaction, this is not the case for the condensate fraction, which embodies both the changes in chemical potential and gap function.

\section{Conclusions}

We have considered the BCS-BEC crossover of a quantum Fermi gas at $T=0$ in the presence of an energy-dependent Fano-Feshbach resonance, driving the system from 


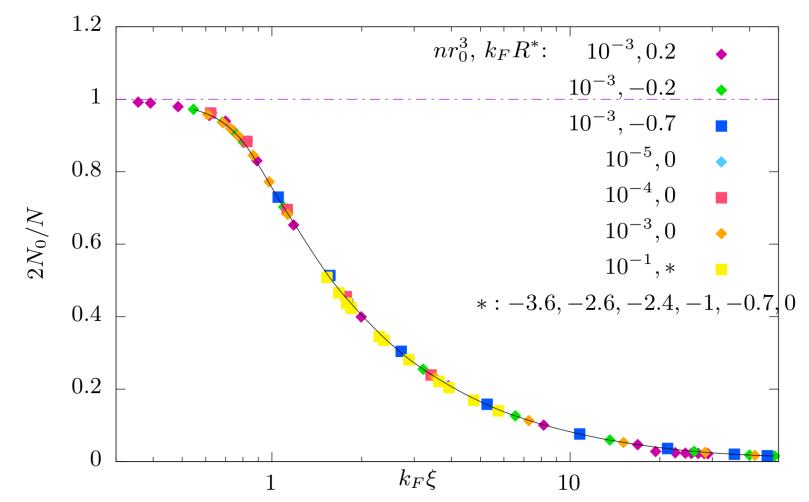

Fig. 6. Condensate fraction $2 N_{0} / N$ vs. $k_{F} \xi$ for the model parameters as in the legend.

broad to narrow limits. We have chosen a minimal microscopic potential reproducing the two-particle resonance physics in terms of the scattering length $a$ and the effective range $R^{*}$ representing the resonance width, and solved the BCS mean-field equations varying $a, R^{*}$ and the density.

We have found that the condensate fraction manifests a universal behavior when the correlation length, measuring the pair size, is used as the crossover parameter, as first introduced in [9]. In addition, the introduction of the negative effective range as a third parameter, stretches the crossover region between the two extreme regimes. As a consequence, while $k_{F} \xi$ remains a suited parameter to describe the crossover, the degree of universal behavior for the chemical potential reduces with respect to the striking result obtained in [9] in a wider range of densities. Remarkably, all the data sets of our three-parameter model for the condensate fraction collapse into a single curve (Fig. 6), embodying both the behavior of the chemical potential and gap function. This result is significant, because it is worked out using a model potential different from $[7,9]$ and stemming from a richer physical mechanism. On the other hand, in the caste literature exploring the BCS-BEC crossover for narrow FanoFeshbach resonances, this result has not been noticed because $k_{F} \xi$, introduced in [9], has not been used to describe the physics. The present work also extends to more negative effective-range values the observable analysis in [28] and, as checked, obtains the same results as those from the two-channel model [33] at corresponding values of the parameters.

In fact, the BCS variational ansatz is not expected to give a complete description, especially around resonance. For example, a comparison performed with Quantum Monte Carlo data available in portions of the parameter space, limited to broad resonances with either zero [26] or small and positive effective ranges [29], evidences the size of beyond mean-field corrections and quantifies the limits of validity of the present mean-field approach. Therefore, the results of the present work may represent a precursor for Quantum Monte Carlo simulations, able to encapsulate the many-particle correlations which here are dropped.

\section{References}

1. S.N. Bose, Zeitschrift fur Physik 26, (1924) 178

2. A. Einstein, Quantentheorie des einatomigen idealen Gases (Preussische Akademie der Wissenschaften, 1924) 
3. J. Bardeen, L.N. Cooper, J.R. Schrieffer, Phys. Rev. 108, (1957) 1175

4. W. Zwerger, The BCS-BEC Crossover and the Unitary Fermi Gas, (Springer Berlin Heidelberg, 2011)

5. D. M. Eagles, Phys. Rev. 186, (1969) 456

6. A. Leggett, J. Phys. Colloq. 7, (1980) C7

7. P. Nozières, S. Schmitt-Rink, J. Low Temp. Phys. 59, (1985) 195

8. Y. J. Uemura, L.P. Le, G.M. Luke, B.J. Sternlieb, W.D. Wu, J.H. Brewer, T.M. Riseman, C.L. Seaman, M.B. Maple, M. Ishikawa, D. G. Hinks, J.D. Jorgensen, G. Saito, H. Yamochi, Phys. Rev. Lett. 66, (1991) 2665

9. F. Pistolesi, G. C. Strinati, G. C., Phys. Rev. B 49, (1994) 6356

10. M. H. Anderson, J.R. Ensher, M.R. Matthews, C.E. Wieman, E.A. Cornell, Science 269, (1995) 5221

11. K. B. Davis, M. Mewes, M.R. Andrews, N.J. van Druten, D.S. Durfee, D.M. Kurn, W. Ketterle, Phys. Rev. Lett. 75, (1995) 3969

12. C. C. Bradley, C.A. Sackett, J.J. Tollett, R.G. Hulet, Phys. Rev. Lett. 75, (1995) 1687

13. F. Dalfovo, S. Giorgini, L.P. Pitaevskii, S. Stringari, Rev. Mod. Phys. 71, (1999) 463

14. M. Greiner, C.A. Regal, D.S. Jin, Nature 426,(2003) 537

15. M.W. Zwierlein, C.A. Stan, C.H. Schunck, S.M.F. Raupach, S. Gupta, Z. Hadzibabic, W. Ketterle, Phys. Rev. Lett. 91,(2003) 250401

16. S. Giorgini, L.P. Pitaevskii, S. Stringari, Rev. Mod. Phys. 80, (2008) 1215

17. U. Fano, Phys.Rev. 124, (1961) 1866

18. H. Feshbach, Ann. Phys.(NY) 19, (1962) 287

19. C. Chin, R. Grimm, P. Julienne, E. Tiesinga, Rev. Mod. Phys. 82, (2010) 1225

20. B. Marcelis, E.G.M. van Kempen, B.J. Verhaar, S.J.J.M.F. Kokkelmans, Phys. Rev. A 70, (2004) 012701

21. T.-L. Ho, X. Cui, W. Li, Phys. Rev. Lett. 108, (2012) 250401

22. V. Gurarie, L. Radzihovsky, Ann. Phys. 322, (2007) 2

23. M. Holland, S.J.J.M.F. Kokkelmans, M.L. Chiofalo, R. Walser, Phys. Rev. Lett. 87, (2001) 120406

24. E. Timmermans, K. Furuya, P. W. Milonni and A. K. Kerman, Phys. Lett. A 285, (2001) 228

25. A. Perali, P. Pieri, and G.C. Strinati, G. C., Phys. Rev. Lett. 93 (2004) 100404

26. G.E. Astrakharchik, J. Boronat, J. Casulleras, S. Giorgini, Phys. Rev. Lett. 93, (2004) 200404

27. S. De Palo, M.L. Chiofalo, M.J. Holland, S.J.J.M.F. Kokkelmans, Phys. Lett. A 327, (2004) 490

28. L.M. Jensen, H.M. Nilsen, G. Watanabe, Phys. Rev. A 74, (2006) 043608

29. M.M. Forbes, S. Gandolfi, A. Gezerlis, Phys. Rev. A 86, (2012) 053603

30. S. J. J. M. F. Kokkelmans, J.N. Milstein, M.L. Chiofalo, R. Walser, M.J. Holland, Phys. Rev. A 65, (2002) 053617

31. J.R. Schrieffer, Theory of Superconductivity (Westview Press, 1999)

32. G. D. Mahan, Many-particle physics, (Springer Science \& Business Media, 2013)

33. L. Salasnich, Phys. Rev. A 86, (2012) 055602 